Universitas, Volumen 2, Número 2, 2008, 29-32, ISSN 2071-2573

(C) 2008 UNAN-León, Editorial Universitaria

\title{
Streptococo del grupo B en mujeres embarazadas atendidas en el Centro de Salud Primero de Mayo. Abril-Agosto 2007
}

\author{
Nancy Dubón Méndez ${ }^{1}$, Marjorie del Socorro Altamirano González¹,Teresa de Jesús Alemán Rivera ${ }^{\star}$
}

1. Estudiantes de IV año de Medicina, Facultad de Ciencias Médicas, Universidad Nacional Autónoma de Nicaragua, León (UNAN-León).

2. Departamento de Microbiología y Parasitología, Facultad de Ciencias Médicas, Universidad Nacional Autónoma de Nicaragua, León (UNAN-León).

\section{RESUMEN}

Streptococos del grupo B (SGB) es una de las principales causas de muertes neonatales en todo el mundo y esta directamente relacionada con la colonización materna al momento del parto. Se realizó un estudio descriptivo de corte transversal que incluyó un total de 120 mujeres embarazadas atendidas en el Centro de Salud 1ro. de mayo-León, con el objetivo de identificar la frecuencia de streptococo del grupo B, debido a que la identificación de esta bacteria influye positivamente en la prevención de infecciones perinatales. La frecuencia de SGB encontrada fue del $11 \%$. El $78 \%$ de las participantes eran amas de casa. En relación con la colonización de las participantes según su edad encontramos que el $36 \%$ de las participantes eran menores de 19 años e iniciaron su vida sexual activa a temprana edad (12-16 años). Además, el $55 \%$ de las participantes colonizadas tenían entre 20-29 semanas de gestación. Los procesos patológicos que presentaron las participantes colonizadas fueron $73 \%$ Leucorrea y $64 \%$ infección de vías urinarias. Los antibióticos de elección para infección por SGB resultaron con una sensibilidad de $77 \%$ penicilina, $62 \%$ clindamicina y $54 \%$ eritromicina; en cuanto a la resistencia antimicrobiana se encontró $92 \%$ Gentamicina y $77 \%$ Oxacilina. El inicio temprano de vida sexual tiene relación con una alta frecuencia de colonización por SGB. Los procesos patológicos que predominaban en las participantes fueron leucorrea e infecciones de vías urinarias. El tratamiento de elección para infección por SGB que es utilizado en la población involucrada no es $100 \%$ sensible.

Palabras claves: Colonización materna, muertes neonatales, resistencia antimicrobiana, Streptococo agalactiae, Nicaragua.

\section{INTRODUCCIÓN}

El Streptococo del grupo B (SGB) o Streptococo agalactiae, es una bacteria Gram positiva que forma parte de la flora genital, catalasa y oxidasa negativa, anaerobia facultativa, cuya colonización en mujeres embarazadas es causa de partos prematuros y bajo peso al nacer, además de ruptura prematura de membranas, abortos espontáneos, infección de vías urinarias y sepsis puerperal. $^{[1,2]}$

La colonización por Streptococos agalactiae en mujeres embarazadas es importante desde el punto de vista clínico, dadas sus posibles implicaciones para el binomio madre-hijo. ${ }^{[2]}$

La infección vaginal y el inicio temprano de vida sexual han sido señaladas como factores que aumentan el riesgo de una mujer de ser portadora de Streptococos del grupo $B{ }^{[3]}$

La infección con SGB afecta a aproximadamente 1 de cada 2.000 bebés nacidos en los Estados Unidos. Cualquier persona puede ser portadora de SGB y entre el $10 \%$ y el $30 \%$ de las mujeres embarazadas lo es. ${ }^{[4]}$
En Latinoamérica en Brasil, México y Venezuela se han observado prevalencias de $18.4 \%, 10.3 \%$ y $32.7 \%$, respectivamente. En otros países en desarrollo se han visto valores menores, India (5.8\%), Libia (5\%) y Arabia Saudita $(13.9 \%) \cdot^{[5,6]}$

Según estudios realizados en el 2005 y 2006 sobre la prevalencia de SGB en mujeres embarazadas en el HEODRA-León fueron de $16.2 \%$ y $8.6 \%$ respectivamente. ${ }^{[1]}$

\section{DISEÑO METODOLÓGICO}

Se realizó un estudio descriptivo de corte transversal, el total de muestras fueron 120 muestras vaginales y 120 muestras rectales que corresponden a todas las embarazadas que asistieron al Centro de Salud $1^{\circ}$ de Mayo en el período comprendido de abril a agosto de 2007 y que cumplieron con los criterios de inclusión.

Para la recolección de datos se utilizó una ficha epidemiológica que contenía variables con los ítems: datos generales, clínicos y factores de riesgos asociadas a nuestros objetivos de trabajo, dicho llenado se hizo a través de una entrevista directa a las participantes. 
Procedimiento de la toma de muestra: Las muestras vaginales y rectales se tomaron con un hisopo estéril y fueron transportadas en AMIES (medio de transporte que permite que la bacteria permanezca viable hasta el momento del cultivo) al Laboratorio de Microbiología de la UNAN-León antes de las 8 horas para procesarlas.

Las muestras vaginales y rectales fueron inoculadas en dos medios: CNA(colítina + acido nalidíxico) y en caldo Todd Hewitt (favorece la recuperación de SGB), recomendado por Center for Disease Control and Prevention (CDC).

Posteriormente se realizó la Tinción de Gram. si resultaba positiva esta prueba (cocos gran positivos en cadenas) se realizaba una prueba de catalasa y una prueba de CAMP ( Factor monosfosfato de adenina cíclica), donde la formación de una zona de hemólisis en forma de flecha indicaba una prueba positiva, si era positiva se realizaba el diagnóstico a través de la prueba de Aglutinación de latéx para identificación de estreptococos grupos ABCDEFG (Lancefield Oxoid, Basingstoke, Hampshire, England).

Para la determinación de la resistencia antimicrobiana se les realizó antibiograma utilizando el método de difusión de discos Kirby Bauer, recomendado por National Committe of Clinical Laboratory Standards de E.E.U.U. ${ }^{[7]}$

Los antibióticos utilizados fueron: ceftriaxone, penicilina, eritromicina, clindamicina, oxacilina, gentamicina y norfloxacina. Luego todas las cepas del SGB se guardaron en leche descremada a $-20^{\circ} \mathrm{C}$ para estudios posteriores. Las variables fueron analizadas en el programa SPSS Versión 13.

\section{RESULTADOS Y DISCUSIÓN}

La frecuencia de Streptococo del grupo B en el Centro de Salud 1ero. de mayo fue del 11\% (ver figura 1); de las cuales el $77 \%$ corresponden a muestras vaginales y el $33 \%$ a muestras rectales, dicho valor se encuentra dentro del rango normal a nivel mundial (5-30\%). Sin embargo, la frecuencia es similar a estudio realizado en México por Ocampo Tórrez (10.3\%) y al realizado en el 2005 en el HEODRA (16.2\%). ${ }^{[1,5]}$

El $36 \%$ de las participantes colonizadas tenían edades menores a 19 años y el 27\% entre 20-25 años, dichos valores son similares a estudio realizado en México por Schuchat que encontraron mayor colonización en mujeres menores de 35 años. ${ }^{[8,9]}$
El nivel de escolaridad de las mujeres embarazadas de mayor frecuencia fue de Secundaria con un $51 \%$. Respecto a la ocupación de las mujeres embarazadas que participaron en nuestro estudio, la mayoría eran amas de casas con un $78 \%$.

Figura 1. Frecuencia de Streptococo del grupo B en mujeres embarazadas, Centro de Salud 10 de Mayo

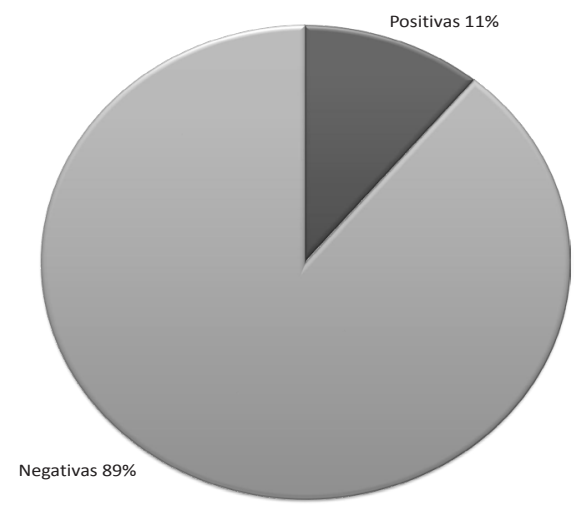

En relación con el número de gestas y la colonización se encontró el $55 \%$ de las embarazadas eran primigestas. EI $55 \%$ de las participantes colonizadas tenían entre 20 29 semanas de gestación (ver figura 2). Dicho valor no lo podemos comparar con otros estudios por que este estudio es el primero que se realiza por que incluimos a toda mujer embarazada sin importar el número de semana de gestación.

También, existe un alto nivel de colonización en embarazadas con menos de 30 semanas de gestación. Esto es de gran importancia ya que si la mujer es colonizada en el primer trimestre del embarazo y esta es tratada adecuadamente con los antibióticos tiende a ser menor el riesgo de estar colonizada al final del embarazo.

En relación a los procesos patológicos y factores de riesgos asociados a la colonización de las participantes se encontró alta frecuencia de Leucorrea $73 \%$ e infecciones de vías urinaria $64 \%$. La presencia de fiebre mayor de $38^{\circ} \mathrm{C}$ y ruptura de membrana no tiene relevancia ya que estos son considerados factores de riesgo al momento del parto y las embarazadas que participaron en nuestro estudio solo llegaron a su visita de control prenatal.

Sin embargo, es de gran importancia conocerlo debido a que la ruptura prematura de membranas puede desencadenar un sinnúmero de procesos mórbidos tanto en la madre como en el recién nacido causándole el inicio temprano de la enfermedad. ${ }^{[10]}$ 
Figura 2. Semanas de gestación versus cultivo por Streptococo del grupo B en mujeres embarazadas, Centro de Salud 10 de Mayo-León 2007

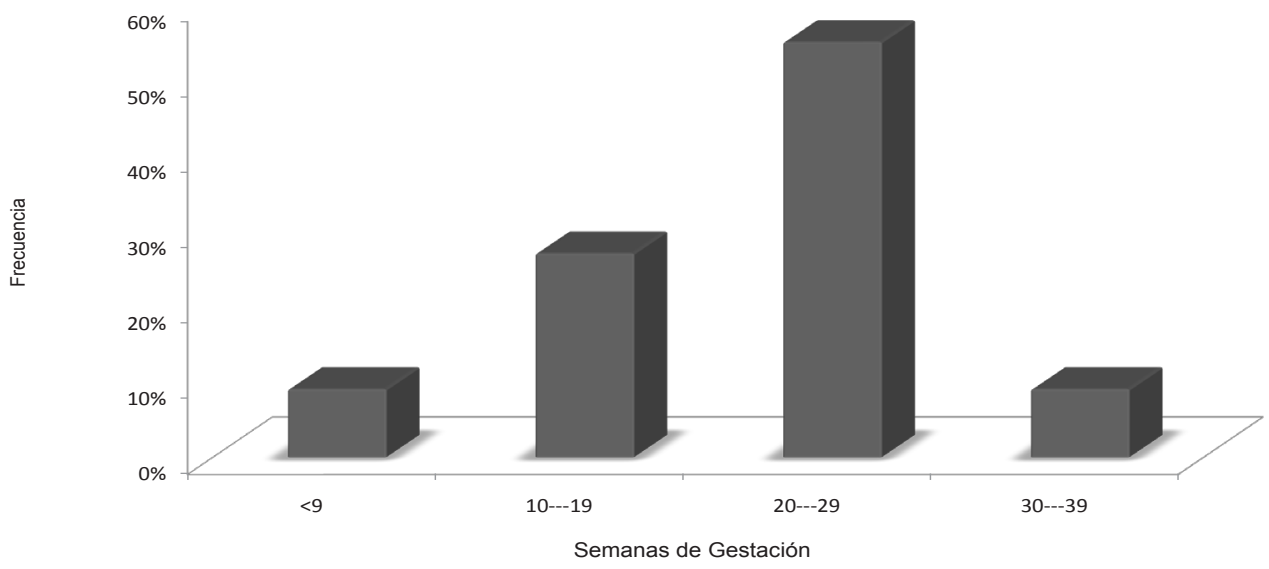

Se encontró que el $55 \%$ de las participantes colonizadas iniciaron su vida sexual activa entre los 12-16 años de edad (ver tabla 1), la literatura señala que el inicio de vida sexual temprana tiene relación con alta frecuencia de colonización por Streptococo del grupo B. ${ }^{[1]}$

Tabla 1. Clasificación de las características organizacionales

\begin{tabular}{cc}
\hline Grupo de edades & Frecuencia Relativa \\
\hline $12-16$ & $55 \%$ \\
$17-21$ & $27 \%$ \\
$22-26$ & $9 \%$ \\
$>27$ & $9 \%$ \\
\hline TOTAL & $100 \%$ \\
\hline
\end{tabular}

Tanto a las muestras vaginales y rectales se les realizó su perfil antimicrobiano obteniendo los siguientes resultados: en cuanto a la sensibilidad: $77 \%$ ceftriaxone, $77 \%$ penicilina, $62 \%$ clindamicina, $54 \%$ eritromicina. En cuanto a la resistencia: gentamicina con un $92 \%$ y un $77 \%$ oxacilina. Un estudio hecho en Barcelona (2002) afirma que existen un incremento de resistencia a Eritromicina (12.45\%), el cual es similar a nuestros resultados Eritromicina (8\%).(ver figura 3)

Esto indica la importancia del trabajo multidisciplinario para evitar la utilización inadecuada de medicamentos en nuestra población a riesgo que son el binomio, madre-hijo, esto porque si la madre esta colonizada, el mismo tipo serotipo de bacteria de SGB será el que colonizará al neonato.

Figura 3. Patrón de sensibilidad y resistencia de las cepas de Streptococo del grupo B, aisladas en las pacientes colonizadas,

Centro de Salud 10 de Mayo-León 2007

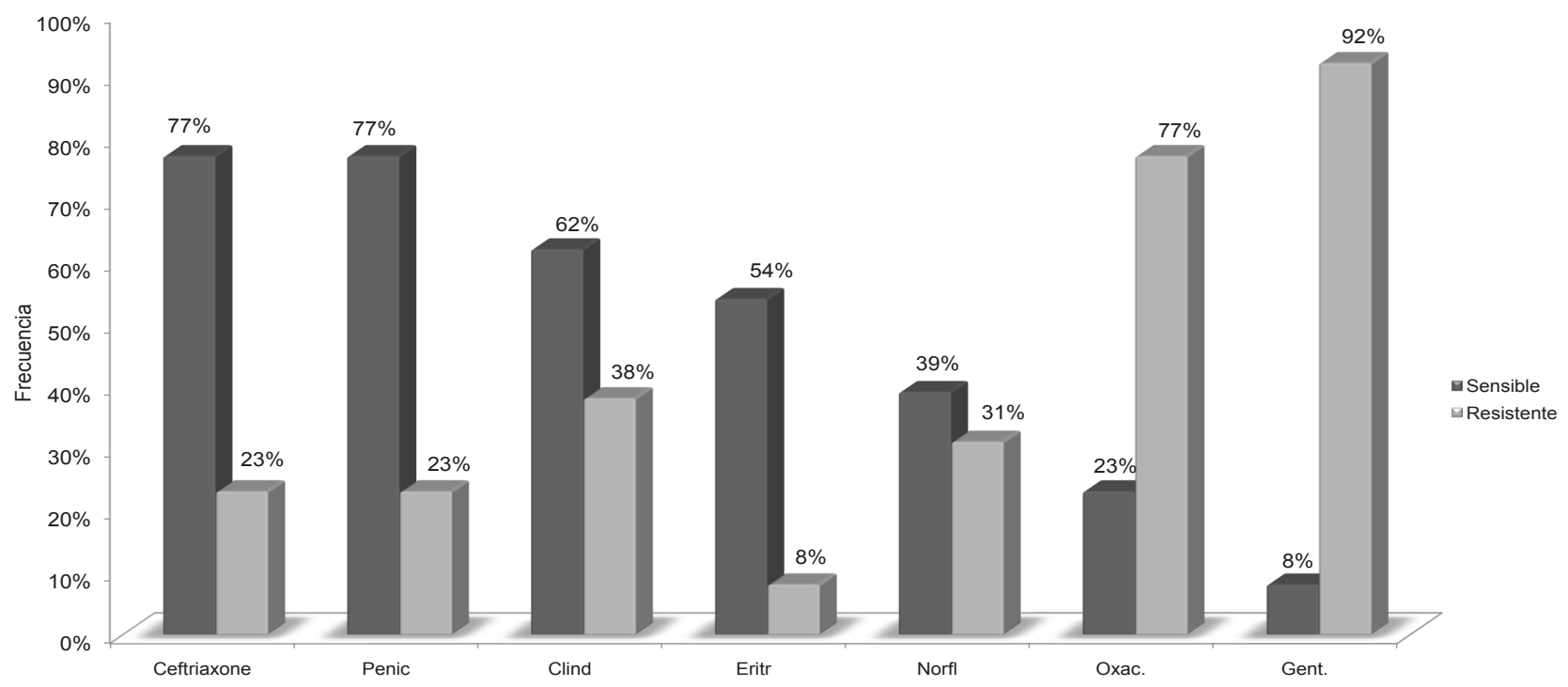


Esto nos lleva a que se aumente la mortalidad perinatal, sino actuamos con esquemas más específicos para estos casos. ${ }^{[11]}$

\section{CONCLUSIONES}

Las infecciones por Streptococo del grupo B continuan siendo una infección frecuente en el embarazo.

El nivel de escolaridad de las participantes era secundaria con un $51 \%$.

El inicio temprano de vida sexual tiene relación con una alta frecuencia de colonización por Streptococo del grupo B.

Las infecciones de vías urinarias asi como la leucorrea se encuentran asociadas con la prfesencia de Streptococo del grupo $B$.

Se encontró alto nivel de resistencia del Streptococos a Gentamicina y Oxacilina, en contraste, la Penicilina y Ceftriaxone confirman siendo los antibioticos de mayor sensibilidad para el manejo de las infecciones.

\section{REFERENCIAS BIBLIOGRÁFICAS}

1. PICADO SÁNCHEZ, O.(2006) Colonización por streptococos $B$ en pacientes con 35-40 semanas de gestación HEODRA-León 2005. Tesis para optar al título de especialista en ginecología y obstreticia. León-Nicaragua, (p. 2; p. 5-12 ;p. 34-36)

2. GALO VARGAS, M. (2003) Comportamiento clínicoepidemiológico del streptococcus del grupo B en neonatos atendidos en el Hospital Oscar Danilo Rosales Argüello y su relación con la sepsis neonatal en el período comprendido julio a diciembre del 2003. Tesis para optar al título de especialidad de Pediatría. León-Nicaragua, (p. 11-25)

3. DI BARTOLOMEO, S., GENTILE, M., PRIORE, G., VALLE, S., DI BELLA, A. (2005) "Streptococcus agalactiae en embarazadas, prevalencia en el hospital Nacional Alejandro Rodales". Revista Argentina No. 3, Vol. 37, (p. 142-144)

4. CRESPO ORTIZ, M., VÉLEZ, J. (1996) “Importancia clínica del streptococos agalactiae como causante de infección". Revista Médica de la Universidad del Valle No.2, Vol. 27, (p. 27:53-89)
5. OCAMPO M; TÓRREZ M. et al. (2000) "Factores asociados, la colonización por streptococo del grupo b en mujeres embarazadas en los altos Chiapas". Salud Pública de Méxic. No. 5, Vol. 42 (p.42-413421).

6. RIERA L., BENAVIDES MORILLO N. (1993) "Colonización por Streptococcus grupo b en embarazadas a término y recién nacidos en una comunidad de Venezuela". Revista de la Sociedad Venezolana de Microbiología Vol. 22 (p. 11: 29597)

7. Clinical and Laboratory Standards Institute. (2005) "Perfomance Standars for antimicrobial susceptibility testing". Fifteenth informatiol supplement No. 1. Vol. 25 (p. 130-132)

8. RIVAS C., TALLAC I., ETCHENIQUE A. (2006) "Colonización vaginorrectal por Streptococos del grupo $B$ en mujeres embarazadas entre las 35 a 37 semanas de gestación". Revista Médica del Uruguay No. 2. Vol. 22 (p. 22:191-196)

9. SCHUCHAT A., DEAVER R. et al. (1994) "Multistate case-control study of maternal risk factors for neonatal group B streptococcal disease". The Pediatric infectious disease journal (p. 13: 623629)

10. ORTIZ L. (2005) Colonización por streptococos B en pacientes con 35-40 semanas de gestación HEODRA-León 2002-2004. Tesis para optar al título de especialista en ginecología y obstreticia. León, Nicaragua, (p. 6-13)

11. GONZÁLEZ A. J., ANDREU A. (2004) "Sensibilidad a antimicrobianos del streptococo del grupo B de transmisión vertical". Enfermedades Infecciosas y Microbiología Clínica. Hospital Vall d'Hebron. Barcelona, España. (p. 286-291) 